Article

\title{
The Magnitude and Waveform of Shock Waves Induced by X-ray Lasers in Water
}

\author{
Claudiu Andrei Stan 1,2,*®D, Koji Motomura ${ }^{3}$, Gabriel Blaj ${ }^{4}{ }^{\oplus}$, Yoshiaki Kumagai ${ }^{3}{ }^{\circledR}$, Yiwen Li $^{3}$, \\ Daehyun You ${ }^{3}{ }^{\circledR}$, Taishi Ono ${ }^{3}$, Armin Kalita ${ }^{1}$, Tadashi Togashi ${ }^{5}$, Shigeki Owada ${ }^{5}$, \\ Kensuke Tono ${ }^{5}$, Makina Yabashi ${ }^{6}$ (D), Tetsuo Katayama ${ }^{5}$ and Kiyoshi Ueda ${ }^{3,6, *}$ \\ 1 Department of Physics, Rutgers University-Newark, Newark, NJ 07102, USA; armin.kalita@rutgers.edu \\ 2 Stanford PULSE Institute, SLAC National Accelerator Laboratory, Menlo Park, CA 94025, USA \\ 3 Institute of Multidisciplinary Research for Advanced Materials, Tohoku University, Sendai 980-8577, Japan; \\ motomura@spring8.or.jp (K.M.); kumagai@go.tuat.ac.jp (Y.K.); yiwen.li@chem.ox.ac.uk (Y.L.); \\ daehyun@dc.tohoku.ac.jp (D.Y.); bebubebubebuchi0love@gmail.com (T.O.) \\ 4 Technology Innovation Directorate, SLAC National Accelerator Laboratory, Menlo Park, CA 94025, USA; \\ blaj@slac.stanford.edu \\ 5 Japan Synchrotron Radiation Research Institute (JASRI), 1-1-1 Kouto, Sayo, Hyogo 679-5198, Japan; \\ tadashit@spring8.or.jp (T.T.); osigeki@spring8.or.jp (S.O.); tono@spring8.or.jp (K.T.); \\ tetsuo@spring8.or.jp (T.K.) \\ 6 RIKEN SPring-8 Center, 1-1-1 Kouto, Sayo, Hyogo 679-5148, Japan; yabashi@spring8.or.jp \\ * Correspondence: claudiu.stan@rutgers.edu (C.A.S.); kiyoshi.ueda@tohoku.ac.jp (K.U.)
}

Received: 3 February 2020; Accepted: 18 February 2020; Published: 22 February 2020

\begin{abstract}
The high energy densities deposited in materials by focused X-ray laser pulses generate shock waves which travel away from the irradiated region, and can generate complex wave patterns or induce phase changes. We determined the time-pressure histories of shocks induced by X-ray laser pulses in liquid water microdrops, by measuring the surface velocity of the microdrops from images recorded during the reflection of the shock at the surface. Measurements were made with $\sim 30 \mu \mathrm{m}$ diameter droplets using $10 \mathrm{keV} \mathrm{X-rays,} \mathrm{for} \mathrm{X-ray} \mathrm{pulse} \mathrm{energies} \mathrm{that} \mathrm{deposited} \mathrm{linear} \mathrm{energy} \mathrm{densities}$ from 3.5 to $120 \mathrm{~mJ} / \mathrm{m}$; measurements were also made with $\sim 60 \mu \mathrm{m}$ diameter drops for a narrower energy range. At a distance of $15 \mu \mathrm{m}$ from the X-ray beam, the peak shock pressures ranged from 44 to $472 \mathrm{MPa}$, and the corresponding time-pressure histories of the shocks had a fast quasi-exponential decay with positive pressure durations estimated to range from 2 to $5 \mathrm{~ns}$. Knowledge of the amplitude and waveform of the shock waves enables accurate modeling of shock propagation and experiment designs that either maximize or minimize the effect of shocks.
\end{abstract}

Keywords: X-ray lasers; shock waves; laser ablation

\section{Introduction}

The deposition of energy into materials using pulsed radiation sources has many practical applications including laser surgery [1,2], laser micromachining [3-5], and chemical synthesis [6]. Besides heating and ablation of the irradiated material, the energy deposited can lead to generation of shock waves if the time scale of energy deposition is shorter than the time in which a sound wave propagates across the irradiated region [1]. These shock waves can carry more than half of the energy deposited by pulsed radiation $[7,8]$ and can lead to unwanted material damage beyond the irradiated region [2]. At the same time, the shock waves produced by pulsed radiation sources are a valuable scientific tool for studying the properties and transformations of materials at high pressures [9-11] and under large tensions [12-17]. 
The development of X-ray free-electron laser facilities (XFEL) operating in the keV photon energy regime $[18,19]$ opened new possibilities for depositing large energy densities in materials. Very high intensities $\left(10^{20} \mathrm{~W} / \mathrm{cm}^{2}\right)$ can be achieved with XFEL pulses because X-rays can be focused more tightly than visible light [20], and the femtosecond duration of the pulses allows the creation of high energy densities, of nonequilibrium states, and the observation of nonlinear X-ray interactions with matter [21-23].

Given the high intensity and the femtosecond duration of XFEL pulses, they are expected to launch shock waves. In samples with thicknesses much smaller than the X-ray absorption length, the shock waves will have a cylindrical symmetry. Such shocks were observed using time-resolved optical imaging, initially in water microjets [24] and afterwards in liquid microdroplets [17]. The shocks launched by XFEL pulses may damage soft samples, such as protein crystals carried in liquid jets for serial femtosecond crystallography [25], in experiments performed at XFELs that generate $\mathrm{MHz}$ pulse repetition rates. While the initial experiments carried with $1.1 \mathrm{MHz}$ pulse trains at the European XFEL [26] did not observe shock damage in protein crystals [27-29], it remains possible that shock damage will occur if such experiments are conducted at the maximum possible pulse rate of 4.4 MHz [30].

The reflection of XFEL shocks at the surface of liquid jets and drops was observed to lead to spallation in droplets [17] and to the generation of shock trains in jets [24,30]. In both cases these phenomena had features not observed in similar experiments with pulsed optical lasers, including the generation of very large negative pressures in drops [17], and the generation of very large sound intensities in jets [30]. The quantitative characterization of these phenomena had large uncertainties or significant noise, which was caused in part by incomplete knowledge of the properties of the initial shock launched by the XFEL pulse, such as the shock waveform.

Here, we report a more precise characterization of shock waves induced by hard XFEL pulses in water, based on measurements of the surface velocity of water microdrops during the reflection of the shock. Improvements in the experimental design allowed us to resolve and measure the decay of the shock pressure after the shock discontinuity, while covering a range of one and a half orders of magnitude in the XFEL pulse energy.

\section{Materials and Methods}

$X$-ray free-electron laser (XFEL) parameters. The experiment was carried out at the SACLA XFEL [19], in the EH4c experimental station on the BL3 beamline [31]. The XFEL pulses had $9.991 \pm 0.029 \mathrm{keV}$ photon energy and an average pulse energy at the XFEL source around 400-500 $\mu \mathrm{J}$. The X-ray beam was focused by a set of Kirkpatrick-Baez (KB) mirrors [32] to a full width half maximum (FWHM) size of $1.3 \mu \mathrm{m}$ horizontally and $1.235 \mu \mathrm{m}$ vertically.

The experiment also used synchronized femtosecond optical pulses for imaging. The imaging pulses were generated by the SACLA laser system (a Ti:sapphire chirped pulse amplifier system with $30 \mathrm{fs}$ duration and $800 \mathrm{~nm}$ wavelength) and were delivered to the EH4c experimental station. The light pulses were frequency doubled to $400 \mathrm{~nm}$ to improve the optical resolution and had an energy of $450 \mu \mathrm{J}$ at the experimental setup.

Experimental setup for measuring the shock wave characteristics. The shock waves were generated by the focused X-ray laser pulses in spherical water microdrops with 29.9-30.5 $\mu \mathrm{m}$ and $61 \mu \mathrm{m}$ diameters, and the shock pressures were calculated from the velocity of the droplets' surface during the reflection of the shock wave. Figure 1a shows the experimental geometry: The center of the droplets was aligned with the XFEL beam to better than $1 \mu \mathrm{m}$, and multiple droplets were first exposed to XFEL pulses and then imaged optically using pulsed laser illumination, to determine how the equatorial diameter of the drops varied as a function of the time delay. Differentiation of the diameter data with respect to the time delay provided the velocity of the droplet's surface in the equatorial region, where the shock waves were reflected at an angle of incidence close to $90^{\circ}$, leading to an instantaneous surface velocity equal to twice the instantaneous particle velocity in the shock wave [33]. The surface position as a 
function of the time delays represents the primary data recorded during the experiment, and the shock properties were calculated from it.
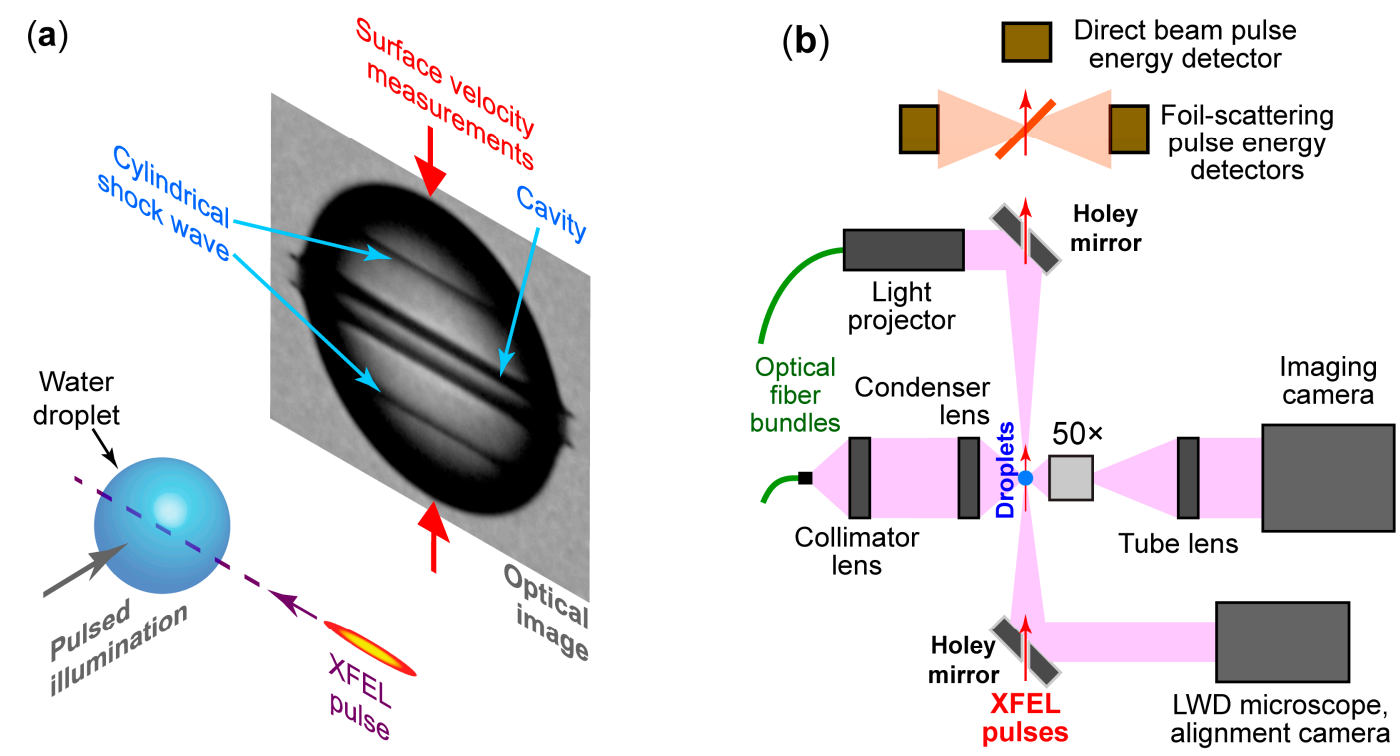

Figure 1. (a) Experimental design. X-ray laser pulses focused to $\sim 1 \mu \mathrm{m}$ passed through the center of $\sim 30 \mu \mathrm{m}$ or $\sim 60 \mu \mathrm{m}$ water microdrops, and launched shock waves. The drops were imaged optically after the X-ray free-electron laser (XFEL) pulse. The photo shows the shock wave in a 30.5- $\mu \mathrm{m}$ diameter drop, $2.29 \mathrm{~ns}$ after the arrival of a $41.5 \mu \mathrm{J}$ XFEL pulse. To characterize the shock waves, the surface velocity of the drop was measured using multiple droplet images over a range of imaging delays. (b) Experimental setup. In addition to the main imaging system with a 50× objective, a lower resolution system was used for droplet alignment. The pulse energy was measured after the droplets for each pulse.

A top-view schematic of the experimental setup, which was operated at ambient temperature and pressure, is shown in Figure 1b. After the X-ray pulses were reflected by the KB focusing mirrors, they exited the beamline through three beryllium and one Kapton thin windows, and traveled a $215 \mathrm{~mm}$ long path in air before being focused. The droplets were overlapped with the beam in the focal plane of the X-rays and were imaged by a high-resolution microscope with a long working distance objective (Mitutoyo M Plan Apo 50×, NA = 0.55). The imaging axis was horizontal and perpendicular to the XFEL beam. An additional low-resolution imaging system was built on the same axis as the XFEL beam, using mirrors with $\sim 3 \mathrm{~mm}$ diameter holes for the XFEL beam, and was used in conjunction with the high-resolution microscope to align the droplets to the beam before each run. After passing through the droplet, the energy of the spent XFEL pulse was measured for each pulse by two detectors that collected X-rays scattered from a Kapton foil, and by a third camera-type detector that also absorbed and blocked the beam.

The water droplets were generated by tapered micronozzles (MJ-AT series, MicroFab Inc., Plano, TX, USA) with 15 and $30 \mu \mathrm{m}$ diameter orifices, equipped with piezoelectric actuators which induced a controlled Rayleigh breakup of the jet into monodisperse droplets. The nozzle control signal was synchronized to the XFEL pulses, to ensure that a droplet was present and properly aligned prior to the arrival of each pulse. The droplet injection was equipped with a temperature control system using circulating liquid from a temperature-controlled bath, which fed heat exchangers for the liquid sample and for a $\sim 1 \mathrm{~cm}$ diameter stream of nitrogen gas coaxial with the nozzle. The temperature control system was not enabled because vibrations from the circulating bath significantly increased the jitter of the drop positions, but the gas stream was still used because it reduced the effect of air drafts in the room on the droplet positions. Thus, the temperature of the whole setup stabilized passively to the temperature of the experimental station and was between 26.1 and $26.25^{\circ} \mathrm{C}$. The temperature was measured with two K-type thermocouples, one placed in contact with the nozzle body, and the other 
in free space, $\sim 1 \mathrm{~mm}$ away from the nozzle orifice; the thermocouples reported temperatures within $0.1^{\circ} \mathrm{C}$ of each other.

The experimental setup and the data collection procedure are similar to the ones used to investigate cavitation and spallation induced in water by X-ray laser pulses [17], and we refer the readers to the previous work for general information on the experiment. Here, we will focus on the upgrades made to that setup and to the data collection and analysis.

Modifications made to the setup to improve the quality of data. The experiments were conducted at atmospheric pressure instead of in a vacuum environment. For the purpose of increasing the accuracy of measurements, operation at atmospheric pressure had multiple advantages: (i) The droplets can be aligned better to the beam since vacuum pump vibrations are eliminated; (ii) the droplet temperature is nearly constant as evaporation, which cools the drops, is much slower at atmospheric pressure than in vacuum; and (iii) it allows better and faster setup and tuning of the optical imaging and of the drop injection, and reduces the duration of the adjustments needed during the beamtime. We note that operation at atmospheric pressure reduces the XFEL intensity through absorption in air, and that shocks from the drops will be partially transmitted into surrounding gas. Nevertheless, these processes did not significantly affect our measurements because the transmission of $\sim 10 \mathrm{keV}$ X-rays through $0.215 \mathrm{~m}$ of air, $88 \%$, was sufficiently large to perform the measurements. Also, the shocks transmitted into the nitrogen gas stream can be neglected, because the ratio of acoustic impedances of liquid water and gaseous nitrogen is $\sim 3000$, therefore the intensity of the shock transmitted into the gas is approximately three orders of magnitude smaller than intensity of the incoming shock [34].

Since the magnitude of shocks depends directly on the XFEL pulse energy, the experiment was designed to accurately measure the pulse energy at the sample, and multiple measurements of the pulse energy were made for each shot. The primary pulse energy measurements were relative, provided by the two photodiodes (Hamamatsu S3590-09) measuring scattering from the Kapton foil placed after the droplets, as shown in Figure 1b. To calibrate the photodiode readings in absolute energy values, a scaling factor was calculated using an absolute energy detector [35] located upstream of the KB focusing mirrors. The absolute detector was apertured to respond only to photons that could be focused by the KB mirrors, and thus provided a measurement proportional to the pulse energy at the sample. The proportionality constant was the beam transmission, which was calculated by compounding losses at the focusing mirror with absorption in air and in the X-ray windows. The absolute energy detector could not be used for all data, since in most of the experimental runs, uncalibrated X-ray attenuators were inserted after the detector.

The absolute detector and the relative detectors used the same type of photodiodes and the same type of electronic reading systems [36]. The shot-to-shot measurements of the absolute detector and the relative detectors were proportional, with a linear correlation coefficient $r=0.9998$. We therefore expect that (i) the relative accuracy of the pulse energy measurements is close to the one of the absolute detectors, which was estimated [36] at $10^{-3}$, and (ii) the absolute accuracy is also close to the one of the absolute detectors, which was measured at $3.6 \%$ at $9.6 \mathrm{keV}$ by comparison with a primary detector standard [37].

To improve the accuracy of measurements of the droplets' surface position, the numerical aperture (NA) of the main imaging system was reduced from the objective specification (NA $=0.55)$ to NA $=0.3$ using irises placed before the condenser lens and in front of the objective lens. The lower NA improved the image contrast and made the edge of the drops perfectly dark (i.e., having a pixel signal of 0 in the camera data), which improved the precision with which the drop surface position was measured.

Data recording and analysis. The data was recorded at an XFEL pulse rate of $30 \mathrm{~Hz}$, in runs that were approximately $20 \mathrm{~min}$ long. During the runs, the time delay of the illumination laser was scanned at a rate of $\sim 10 \mathrm{ps} / \mathrm{s}$, resulting in probing a time delay range of $\sim 12 \mathrm{~ns}$ during a run. The relatively slow scan rate of the time delay resulted in finely spaced time delay data, but it also made the measurements sensitive to slow drifts in the droplet diameter and position. 
The time delay between the XFEL pulse and the illumination of the image could be set statically with 1 ps accuracy. During the time delay scans, we recorded this delay for each XFEL shot using a timer-counter (Keysight 53230A, 20 ps specified accuracy). The timer measured the time delay between the XFEL electronic trigger and the signal from a fast photodiode (Hamamatsu G4176-03) that picked up part of the illumination light. The zero time delay condition between $\mathrm{X}$-rays and illumination was determined before the beamtime using the fast photodiode, and was refined during the beamtime to an accuracy around 2 ps using the onset of the ionization caused by the XFEL beam, at the maximum available intensity.

Since the XFEL pulses had a wide distribution of pulse energies, the data was binned by pulse energy in consecutive logarithmic bins with a $0.05(5 \%)$ relative width, i.e., the data in a bin had pulse energies ranging from 0.95 to 1 times of the bin maximum. From one run, nine to 11 binned data sets were extracted, according to the condition that each binned set had sufficient data to sample the time delays with an average time step of $\sim 10 \mathrm{ps}$.

The drop images had a magnification of $207 \mathrm{~nm} /$ pixel, an optical resolution better than $780 \mathrm{~nm}$, and were recorded with a high-speed camera (Phantom Miro M340, Vison Research). The procedure for determining the droplet diameters is similar to the one described previously [17] with the following differences: (i) We extracted the image data directly from the raw camera data in a compressed 10-bit format and linearized it, instead of converting the data to 8-bit movie format. (ii) Since the raw droplet images already had very good edge contrast, it was not necessary to apply a nonlinear rescaling of the pixel data. Images in which the droplet center was misaligned by more than $\sim 0.5 \mu \mathrm{m}$ from the XFEL beam were removed from further analysis, because for such drops the shock waves arriving at opposite regions along the drop's equator had sufficiently different arrival times to affect further analysis.

The operation in open atmosphere and the upgrades to the optical setup and image processing reduced the jitter of drop diameter measurements below $50 \mathrm{~nm}$ peak-to-peak in eight out of nine runs. This is a several-fold improvement from the previous study on XFEL-induced spallation [17], where the drop diameter data had a jitter ranging from 100 to $250 \mathrm{~nm}$ peak-to-peak.

The noise in the drop diameter measurements prevented a point-by-point derivation of the data with respect to the time delay, as the velocity data would have been extremely noisy. We chose to fit the diameter data with an exponentially decaying function:

$$
D_{D}(t)-\mathrm{D}_{0}(t)=\mathrm{V}_{0}\left(t-\mathrm{t}_{\text {shock }}\right) \exp \left[-\left(t-\mathrm{t}_{\text {shock }}\right) / \mathrm{t}_{\text {decay }}\right],
$$

where $t$ is the time elapsed from the XFEL pulse arrival, $D_{D}(t)$ is the measured diameter of the drop, $D_{0}(t)$ is the diameter of the drop before the shock arrival including a linear correction of the experimental drifts in the drop diameter, $\mathrm{V}_{0}$ is the initial diameter expansion velocity upon the arrival of the shock wave, $t_{\text {shock }}$ is the arrival time of the shock (measured from the XFEL pulse arrival), and $t_{\text {decay }}$ is the time in which the surface velocity decays from its peak value to zero. This diameter function leads to a quasi-exponential decay of the surface velocity. It has three fit parameters $\left(V_{0}, t_{\text {shock }}, t_{\text {decay }}\right)$ that have a clear physical significance and can be quickly estimated from the data to provide starting values for the fitting. Appendix A contains further details about the fit function and the fitting procedure.

The particle velocity in the shock, $u_{p}(t)$, is equal to half of the surface velocity, which is itself half of the droplet diameter expansion velocity. Thus, the particle velocity is one quarter of the diameter velocity and is given by:

$$
u_{p}(t)=(1 / 4) \mathrm{d} / \mathrm{d} t\left[D_{D}(t)-D_{0}(t)\right]
$$

The initial pressure of the shock, $P_{S}\left(t_{\text {shock }}\right)$, was derived using the Rankine-Hugoniot conditions [38] and is given by:

$$
P_{S}\left(\mathrm{t}_{\text {shock }}\right)=\rho_{0} u_{S}\left(\mathrm{t}_{\text {shock }}\right) u_{p}\left(\mathrm{t}_{\text {shock }}\right),
$$

where $\rho_{0}$ is the water density before the shock arrival, and $u_{s}$ is the speed of the shock wave when it arrives at the surface. To calculate the speed of the shock wave we used the empirical relation [39] $u_{s}\left(t_{\text {shock }}\right)=c_{0}+2 u_{p}\left(t_{\text {shock }}\right)$, where $c_{0}$ is the speed of sound in water prior to the shock arrival. 
The pressure of the incoming wave after the shock discontinuity, $P_{S}\left(t>t_{\text {shock }}\right)$, was calculated by numerically integrating of temporal derivative of the pressure, using the relation:

$$
\mathrm{d} P_{S}(t) / \mathrm{d} t=\rho_{0} \mathrm{C}_{\mathrm{C}}\left[P_{S}(t)\right] \mathrm{d} u_{p}(t) / \mathrm{d} t,
$$

where $\mathrm{C}_{C}\left[P_{S}(t)\right]$ is the cylindrical phase velocity [40] in the decaying pressure wave. Equation (4) models the adiabatic evolution of the liquid after the shock discontinuity [40-42] taking into account the cylindrical geometry of the shock [43]. Equation (4) and the calculation of the phase velocity are discussed further in Appendix B.

\section{Results}

To cover a wide range of pulse energies, multiple runs were recorded with XFEL pulses attenuated in different amounts by a combination of thin Si wafers and Al foils. Measurements with 29.9-30.5 $\mu \mathrm{m}$ diameter drops were made for pulse energies ranging from $7.1 \mu \mathrm{J}$ to $243 \mu \mathrm{J}$ at the droplets. Injection of the $61 \mu \mathrm{m}$ diameter drops was affected by drifts in the undisturbed drop diameters, and these drifts were often larger than the measured signal. Only one experimental run with $61 \mu \mathrm{m}$ diameter drops had sufficiently stable drops, and allowed measurements for pulse energies ranging from $47 \mu \mathrm{J}$ to $79 \mu \mathrm{J}$.

Raw data sets extracted from one experimental run are shown in Figure 2a. As the pulse energy increased, (i) the surface displacement became larger, which implies a higher shock pressure, and (ii) shocks arrived at the surface earlier, because the shock velocity increases with the shock amplitude. Figure $2 \mathrm{a}$ also shows the slowly-drifting undisturbed drop diameter $\mathrm{D}_{0}(t)$, calculated by linear fitting of the diameter data just before the arrival of the shock. The drift was less than one thousandth of the drop diameter over the time delays used for measurements (8-10 ns) and the subtraction of $\mathrm{D}_{0}(t)$ from the data was sufficient to correct for the drifts.
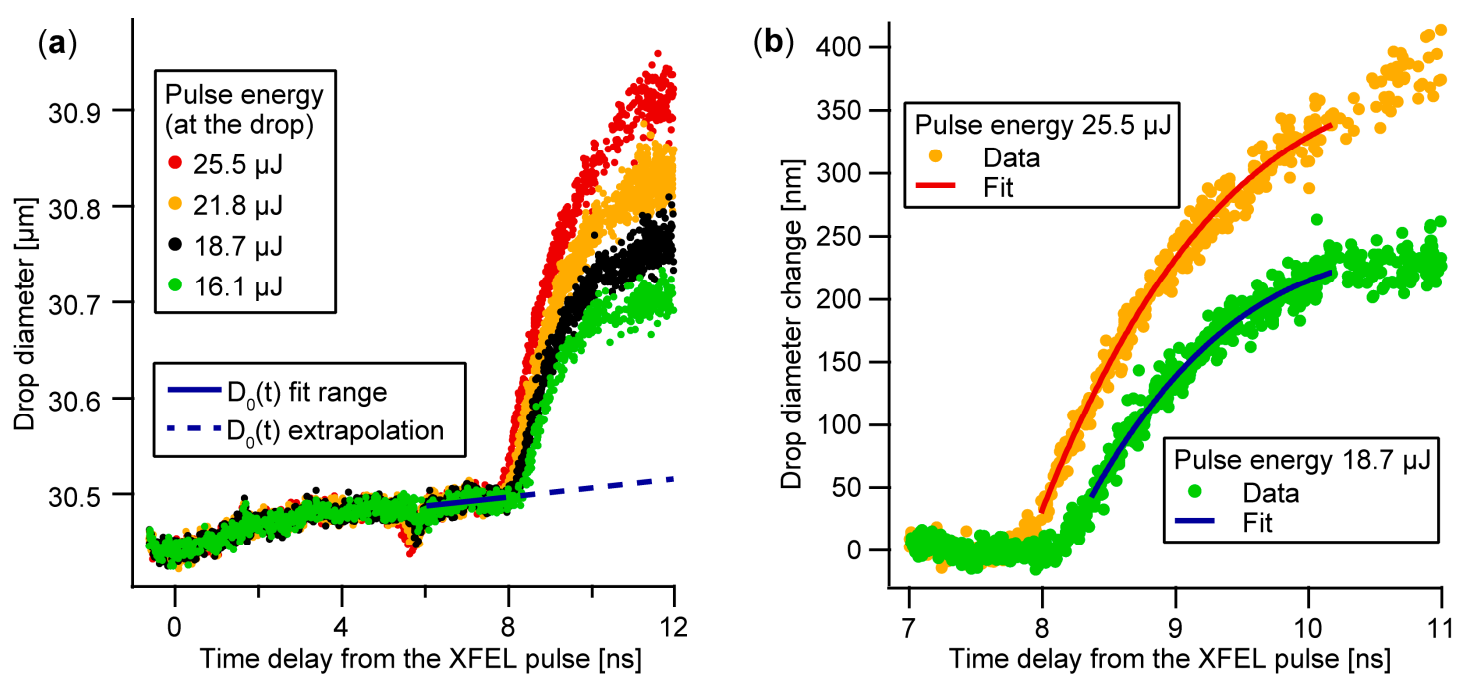

Figure 2. Experimental measurements and the fit of surface motion data. (a) Typical drop diameter measurements from a single experimental run. For clarity, only four of the 10 extracted data sets are shown. Since these data sets were recorded simultaneously, they exhibit the same drift in the undisturbed droplet diameter, $\mathrm{D}_{0}(t)$. (b) The change in drop diameter during the shock reflection, $D_{D}(t)-\mathrm{D}_{0}(t)$, for two of the data sets shown in the left panel, and their analytical fits made using Equation (1). To reduce the influence of shock wave reflections, the data is fitted only up to the delay corresponding to the propagation of pressure waves at the speed of sound.

The fitting of data is illustrated in Figure $2 b$. After subtracting $D_{0}(t)$, the data was fitted in a limited range of time delays, as explained in Appendix A. The fit parameters for all recorded data sets are given in Table A1 in Appendix C, along with the initial shock pressure. Data in Table A1 shows 
that as the pulse energy increased, the initial shock pressure $\left(P_{S}\left(t_{\text {decay }}\right)\right)$ increased, the arrival time of the shock $\left(t_{\text {shock }}\right)$ decreased, and the decay time of the surface velocity $\left(t_{\text {decay }}\right)$ increased.

The dependence of the initial shock pressure (the jump pressure) on the pulse energy and on the linear energy density deposited by X-rays is shown in Figure 3 for all data sets. The linear energy deposited is given by the product of the $\mathrm{X}$-ray pulse energy and the $\mathrm{X}$-ray energy-absorption coefficient ( $493 \mathrm{~m}^{-1}$ at $10 \mathrm{keV}$ for water). At the lowest pulse energies, the relative quality of the fit decreased because the displacement of the interface was approaching the typical jitter and drift of the initial droplet diameter. The reproducibility of the peak pressure measurements, estimated as the largest difference between independent measurements made at the same pulse energies in different runs, was approximately $25 \mathrm{MPa}$.

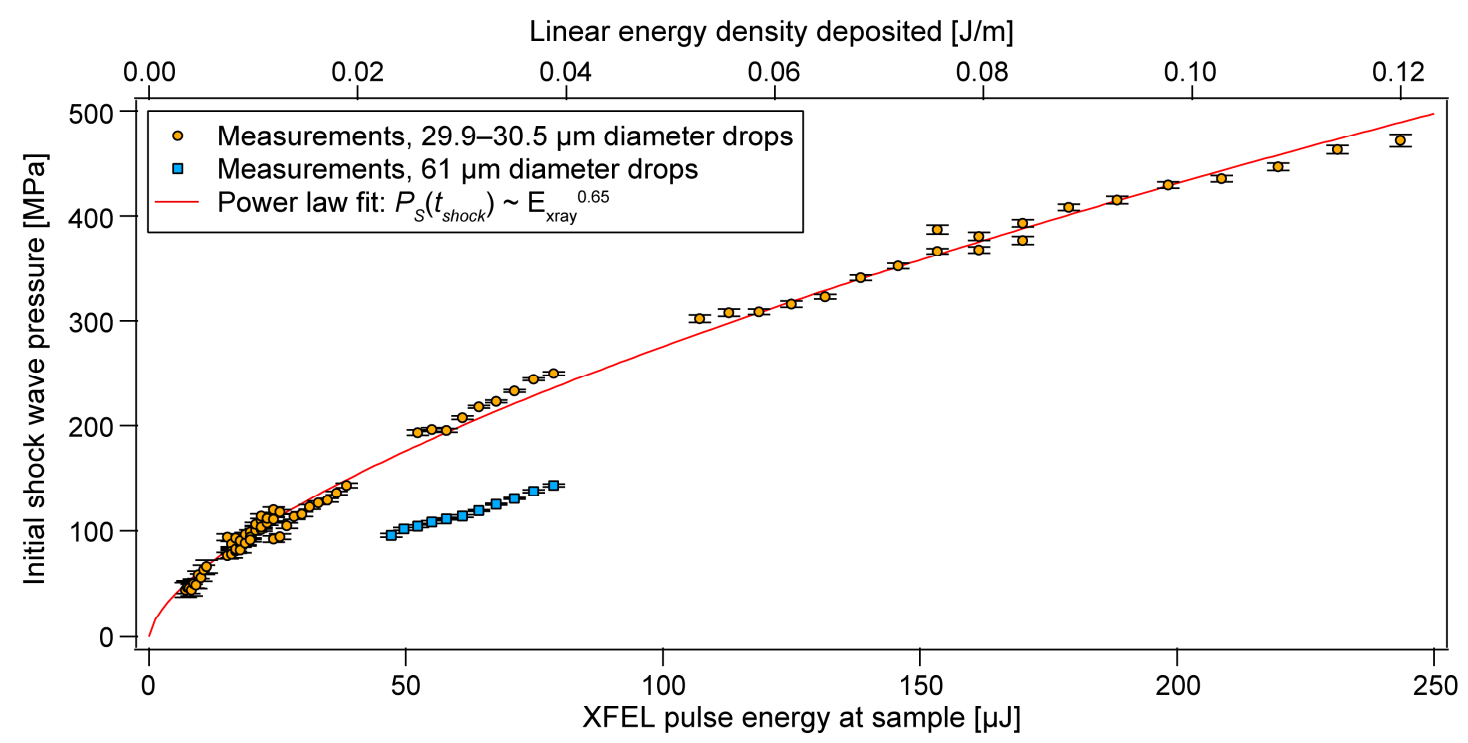

Figure 3. Dependence of the peak shock pressure on the pulse energy. At the same pulse energy, the pressure was smaller in the larger drops due to the geometrical decay of the expanding cylindrical shock. The peak pressures in the small drops were fitted to a power-law function of the pulse energy.

For 29.9-30.5 $\mu \mathrm{m}$ diameter drops, the initial shock pressure can be fit with a power-law dependence on the pulse energy $E_{x r a y}, P_{S}\left(t_{\text {shock }}\right) \sim E_{x r a y} 0.65$. The data for $61 \mu$ m diameter drops does not have sufficient dynamic range in the pulse energies for a power law fit.

The temporal evolution of the incoming shock pressure, at the equatorial surface of the drop, was calculated through integration of Equation (4) and is shown in Figure 4 for a subset of measurements. For approximately the same drop diameters, the pressure waveform was similar for different pulse energies and extrapolated to zero pressure $\sim 1 \mathrm{~ns}$ after the acoustic time, which is earlier than the time delay when the fitted particle velocity crosses zero (i.e., $t_{\text {shock }}+t_{\text {decay }}$ ). This behavior is caused by the phase velocity $C_{C}\left[P_{S}(t)\right]$ being larger than the speed of sound due to the shock decay and the cylindrical geometry, which following Equation (4) leads to a faster relative decay of the pressure, $\mathrm{d} P_{S} / P_{S}$, than the relative decay of the particle velocity, $\mathrm{d} u_{p} / u_{p}$.

We note that our calculation of pressure applies to Lagrangian element of fluid located at the surface (i.e., it applies to the same Lagrangian position). Therefore, due to the motion of the surface, the pressure curves in Figure 4 are slightly different from the pressure evolution at the initial position of the surface (the Eulerian position). Also, due to the conservation of energy in cylindrical symmetry, the temporal waveform of the pressure does not have the same shape as the spatial waveform of the pressure. 

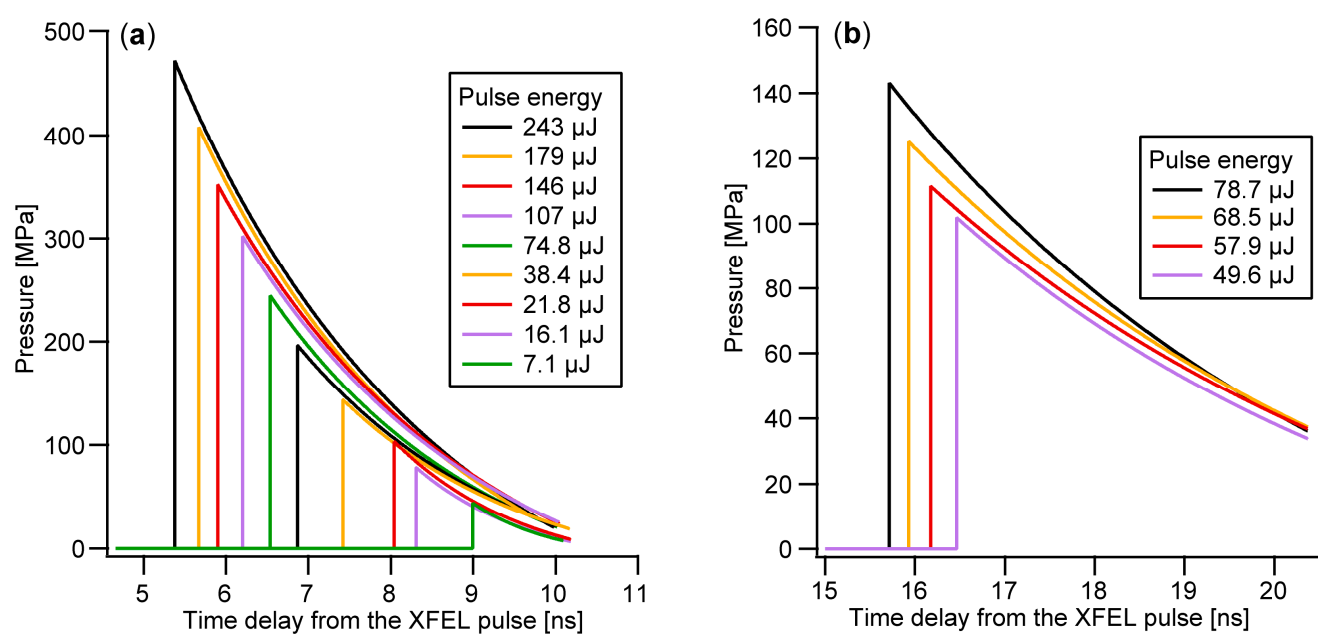

Figure 4. Shock wave waveforms, calculated as described in the text from the fitted surface motion functions. For clarity, the data sets include the shocks with the smallest and largest amplitudes, and a subset of all available waveforms. (a) Waveforms at the surface of small drops, 14.95-15.25 $\mu \mathrm{m}$ from the X-ray beam center. (b) Waveforms at the surface of large drops, $30.5 \mu \mathrm{m}$ from the X-ray beam.

\section{Discussion}

The energy carried by the shock wave. The energy carried by the shock can be calculated by integrating the instantaneous wave intensity, $I_{S}(t)$, which is given by:

$$
I_{S}(t)=P_{S}(t) u_{p}(t)
$$

Because the shocks launched in the droplets are, to a good approximation, cylindrically symmetric, their energies were expressed as linear energy densities. The linear energy density carried by the shock, from $t_{\text {shock }}$ until $t_{\text {acoustic }}$, is shown as a function of the deposited linear energy density in Figure $5 \mathrm{a}$, and the ratio of the shock energy to the deposited energy is shown in Figure 5b. Since the shock wave energies shown in Figure 5 have been integrated only up to $t_{\text {acoustic, }}$, they are less than the total energy carried by the shock. Also, the shocks already dissipated a fraction of their energy via irreversible heating of the liquid, when they had large amplitudes shortly after generation [8]. Nevertheless, the trends in the energy carried by shocks can be useful for designing XFEL experiments. Both the shock energy and the fractional amount of energy increased with the XFEL pulse energy, thus the energy of the shock increased faster than linearly with the pulse energy.
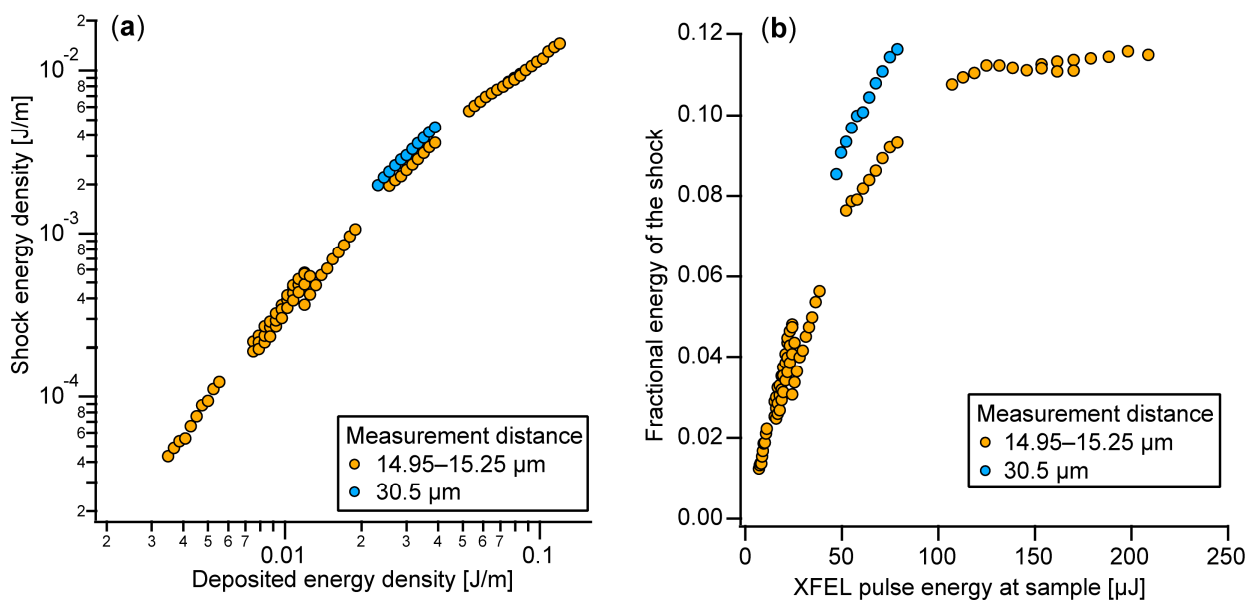

Figure 5. The energy carried by the shock waveforms up to $t_{\text {acoustic }}$ (a) Shock energy as a function of deposited energy. (b) Fraction of the absorbed energy carried by the shock waves. 
Within the range of parameters of our experiments $\left(\mathrm{P}_{\mathrm{S}}<500 \mathrm{MPa}\right)$, the shock waves were weak, in the sense that the energy lost by increasing the entropy of the shocked water remained small relative to the total energy of the shock [44]. We thus expect that the energy of the shock decayed slowly as it traveled between positions corresponding to the radii of the small and large droplets, and Figure 5 shows that at the same XFEL pulse energy, the calculated energy was approximately the same for $\sim 30 \mu \mathrm{m}$ and $61 \mu \mathrm{m}$ diameter drops. The larger energy calculated for the $61 \mu \mathrm{m}$ drops can be explained by the experimental uncertainty in energy, which arises from the uncertainty in pressure. We note that the maximum conversion ratio of absorbed energy to shock energy shown in Figure $5 b, \sim 12 \%$, falls in between the conversion ratios observed for near-field and far-field shock waves generated by pulsed optical ablation in water $[7,8]$.

The short duration of the shock wave. Two important characteristics of the cylindrical shocks generated by XFEL pulses in droplets are their brief duration and the likely decay of pressure to negative values (tension). The pressures in Figure 4a decayed to less than one tenth of their peak pressure by $t_{\text {acoustic }}$. Since in our experiment the pressure decayed faster than the particle velocity, shocks that led to a maximum in drop diameter, and thus to the particle velocity crossing zero (see the example in Appendix A), had a negative pressure phase. Extrapolation of the waveforms in Figure 4a indicates that the pressure reached zero between 2 and $5 \mathrm{~ns}$ after the shock arrival, depending on the pulse energy. Multiplying these durations with the speed of sound in water $(\sim 1500 \mathrm{~m} / \mathrm{s})$ we can estimate the approximate length of the positive waveform $(3$ to $7.5 \mu \mathrm{m})$, which is smaller than the $15 \mu \mathrm{m}$ radius of the drop. Because the positive part of the shock waveform was shorter than the drop radius, its reflections traveled inside the liquid until they no longer overlapped with the positive pressure phase of the incoming shock. Since the shock reflections at a water-air interface are rarefaction waves [34], pronounced pressure oscillations should occur in jets and drops exposed to XFEL pulses. Two previously observed effects of these pressure oscillations are spallation in droplets [17] and the formation of shock trains in jets $[24,30]$.

Recent numerical simulations of experiments on microscale ablation in water [16,45-47] allow us to compare qualitatively the waveform of shocks produced by XFEL pulses and by optical laser pulses. To simulate the shock dynamics and match it to the experimental conditions, proper initial conditions need to be defined. A closed spherical bubble with $8 \mu \mathrm{m}$ diameter and 6 GPa pressure was used for an optical ablation experiment $[16,45]$, and an open cylinder with $0.5 \mu \mathrm{m}$ diameter and $100 \mathrm{GPa}$ pressure was used for an XFEL experiment [47]. In the case of optical ablation with spherical symmetry, the calculated shock waveforms were relatively long and did not reach zero pressure. In contrast, the simulation of the XFEL spallation experiment in drops predicted short waveforms with a negative pressure phase. Short-lived shocks that decay to approximately zero pressure were also predicted in simulations of laser ablation in a ring pattern, in a quasi-2D geometry [46]; in this case the excited region was chosen to have a width of $1.6 \mu \mathrm{m}$ and an initial pressure of $2 \mathrm{GPa}$, and the simulation did not include the formation of a gas bubble in the excited region. Overall, these simulations suggest that the generation of nanosecond shock waveforms that decay to zero pressure requires that the region where the energy is absorbed is at most a few microns in size, and that the ablation does not lead to high-pressure gas trapped inside bubbles.

Cavitation and spallation. Cavitation and spallation were observed in additional experimental runs that probed time delays longer than the ones we used here for shock wave analysis. Qualitatively, these phenomena were the same as the ones reported previously [17], but quantitative properties such as the arrival time of the spall wave were different, because they depend on the droplet diameter and on the pulse energy. The present experimental setup should enable measurements of the cavitation pressures with improved accuracy and over a wider range of experimental parameters, because the raw data was less noisy and more accurate than the data previously recorded in vacuum. Our present work also indicates the necessity to consider how the short duration of the shock waveform affects the spall dynamics [48]. For example, simulations of XFEL-induced shocks in drops showed an increase in the rise time of the reflected wave as it traveled [47]. An increase in the rise time of the negative 
pressure pulse will lower the strain rate at which the liquid is stretched, and can lower the peak tension of the negative pressure wave.

Applicability and accuracy of pump-probe optical microscopy for characterization shock waves at the microscale. The small size of the liquid droplets and their high surface curvature limits the range of techniques that can be used to characterize shock wave properties. For example, single-shot interferometric techniques such as the velocity interferometer system for any reflector (VISAR) [49] cannot be applied to microdrops because interferometry requires a sufficiently flat surface over the area probed by the light beam, such that the reflected light has an approximately constant phase. Currently, the minimum beam spot size of commercial interferometers, $\sim 100 \mu \mathrm{m}$, is larger than the drops we used. The curvature of the droplet's surface makes them well suited, however, for methods based on optical imaging. Even a transparent drop can have a high-contrast dark edge, if the numerical aperture is sufficiently small. Spherical surfaces have their maximum lateral extent at a well-defined position along the optical axis, and if this position is in focus, a sharp image of the surface can be obtained.

Our results show that a temporal resolution of at least $0.1-1 \mathrm{~ns}$ is needed to resolve the shock waveform in microdrops. This temporal resolution would require a very high-speed framing camera to record the surface velocity in a single shot. Framing cameras were used successfully to resolve the dynamics of underwater explosions in a millimeter-sized system [50], but for micron-sized drops such cameras must be able to operate at $\mathrm{GHz}$ frame rates, which is close to the state of the art for commercial systems. Nevertheless, since liquid micron-sized droplets can be generated reproducibly at high rates, pump-probe techniques can be used instead. To our knowledge, outside the XFEL droplet ablation experiments, the pump-probe measurement of surface velocities in microscale liquid systems was applied in a study of cavitation in an optofluidic chip [51], but in that case the surface position was measured only once, $40 \mathrm{~ns}$ after the shock arrival, therefore the shock waveforms could not be determined.

The accuracy of our shock characterization method is primarily limited by the reproducibility of the drop diameters. At short time scales, this uncertainty arises from the jitter in drop diameter measurements, which typically was below $50 \mathrm{~nm}$ peak to peak. For a determination of surface velocity within a $1 \mathrm{~ns}$ interval, this corresponds to a $50 \mathrm{~m} / \mathrm{s}$ uncertainty in the droplet expansion velocity (or $12.5 \mathrm{~m} / \mathrm{s}$ for the particle velocity), which in turn corresponds to an uncertainty of approximately $20 \mathrm{MPa}$ in the shock pressure. Nevertheless, this type of uncertainty can be reduced by averaging multiple measurements. At longer time scales, the magnitude and sign of the slow drifts in the unperturbed droplet diameter can become important. In this case the overall accuracy can be estimated as half the spread of $\mathrm{V}_{0}$ data in Table $\mathrm{A} 1$, for measurements made at the same energy but in different runs; the uncertainty of surface velocity is approximately $25 \mathrm{~m} / \mathrm{s}$, one order of magnitude worse than commercial VISAR instruments.

The cylindrical shock waves produced by hard X-ray laser pulses in liquid water can be detrimental or beneficial in different types of XFEL studies, and the ability to predict their properties, including their waveforms, is useful in both cases. The brief duration of XFEL-generated shocks could prove useful for subjecting samples to large pressures that last only $\sim 1 \mathrm{~ns}$, which would allow, for example, to initiate a dynamic transformation in a material but not allow it to complete. Here we provided comprehensive amplitude and waveform data for shocks propagating in water and passing through a surface $15 \mu \mathrm{m}$ away from the beam's center, but the method can be applied to other liquids and propagation distances. Also, our experimental data could be used as a benchmark for future analytical or numerical models that might predict the properties of the XFEL-induced shocks for any propagation distance and any energy of the XFEL pulses.

Author Contributions: C.A.S. conceived the experiment; C.A.S. and K.U. coordinated the experimental team; K.M., T.K., C.A.S., K.T., S.O., T.T. and M.Y. set up the experiment; S.O. and T.T. prepared the femtosecond optical pulses for imaging liquid droplets; K.M., G.B., Y.K., Y.L., D.Y., T.O., T.K., K.U. and C.A.S. conducted the experiment; C.A.S., G.B., and A.K. analyzed the data; C.A.S. interpreted the data and wrote the paper. All authors have read and agree to the published version of the manuscript. 
Funding: The experiments were supported by the U.S. Department of Energy, Office of Science, Chemical Sciences, Geosciences, and Biosciences Division, by the X-ray Free Electron Laser Priority Strategy Program of the MEXT, by the Research Program of 'Dynamic Alliance for Open Innovation Bridging Human, Environment and Materials" in "Network Joint Research Center for Materials and Devices,' and by the IMRAM project. Data analysis and interpretation were primarily supported by startup funds from Rutgers University-Newark.

Acknowledgments: We thank the staff of SACLA for their support and dedicated contributions, and Howard Stone for helpful discussions. The XFEL experiments were performed at the BL3 of SACLA with the approval of the Japan Synchrotron Radiation Research Institute (JASRI; Proposal No. 2016B8016).

Conflicts of Interest: The authors declare no conflict of interest. The funders had no role in the design of the study; in the collection, analyses, or interpretation of data; in the writing of the manuscript, or in the decision to publish the results.

\section{Appendix A}

Empirical fitting of the droplet diameter data with an analytical function. Since the experimental droplet diameter data was recorded from independent single-shot measurements, it included experimental noise in the range of 20 to $50 \mathrm{~nm}$ peak-to-peak for undisturbed drops. Also, since the time delays were sampled at $\sim 10$ ps or less, but the delay data was recorded with a timer-counter with $\sim 20$ ps standard deviation, the delay measurements also had significant shot-to-shot noise. This degree of experimental noise precluded a direct differentiation of diameter data to extract surface velocities. Although shock kinematics data can in principle be calculated from the raw data using Savitzki-Golay fitting [17,52], here we chose to fit the raw data using an analytical function because it provided a more compact result and the fit quality was good across all data.

The fit function, which is given by Equation (1), is the integral of the Friedlander function $[53,54]$ : $(1-z) \exp (-z)$, with $z>0$ and $z=\left(t-t_{\text {shock }}\right) / t_{\text {decay }}$. The Friedlander function represents a waveform with a discontinuous pressure jump at $z=0$, followed by an immediate decay that lowers the pressure to a minimum that is below the initial pressure. The Friedlander function was found to describe accurately the pressure-time history from hemispherical blast waves in air [54], and here we implicitly assumed that this function can also describe with sufficient accuracy the particle velocity-time history for the cylindrical shocks in water.

To fit the data with Equation (1), we first subtracted from the raw data the drop diameter prior to the arrival of the shock, $\mathrm{D}_{0}(t)$, which we expressed as linear function of time because it drifted during the experimental runs. The fit procedure is illustrated in Figure 2, and Figure A1 shows the same procedure for a different experimental run. The run shown in Figure A1 had the largest drift in $\mathrm{D}_{0}(t)$ among all measurements presented here. Nevertheless, it was still possible to correct the data for the drifts because the relative change in the droplet diameter over the fitted time delays was less than 0.002 , which is smaller than the 0.02 relative spread of undisturbed drop diameters among the runs using the smaller drops (29.9-30.5 $\mu \mathrm{m}$ diameters).

Variations in the drop diameter between different runs can affect the merging of different data sets because in a larger drop the shock will arrive later, and will have a lower pressure, than in a smaller drop. Nevertheless, for our measurements the drifts and the run-to-run variations in drop diameters had only a small effect on the accuracy of the derived data. For example, Figure 3 shows that the peak pressure scales by less than the inverse of the propagation distance, therefore the relative systematic error in the peak pressure, caused by a slightly different droplet diameter, will be smaller than the relative differences $(\sim 0.02)$ between droplet diameters in different runs.

Using surface velocity measurements in droplets, the properties of the cylindrical shock generated by XFEL pulses can be determined directly only up to a limited time delay, because two additional types of pressure waves can arrive at the surface and overlap with the cylindrical shock: (i) Oblique shock reflections from surface regions away from the equatorial zone, and (ii) if spallation occurs, reflections from the surface of the void generated by spallation. To minimize the chance of measuring the contribution of additional waves, the data was fitted only up to either (i) the 'acoustic' time delay $t_{\text {acoustic }}$, which is equal to the time in which a low-amplitude wave propagates at the speed of sound 
from the center to the equator of an undisturbed drop, or (ii) until the arrival of the spallation reflection, if earlier than the acoustic time. Also, the shock waveforms were computed only up to the acoustic time.
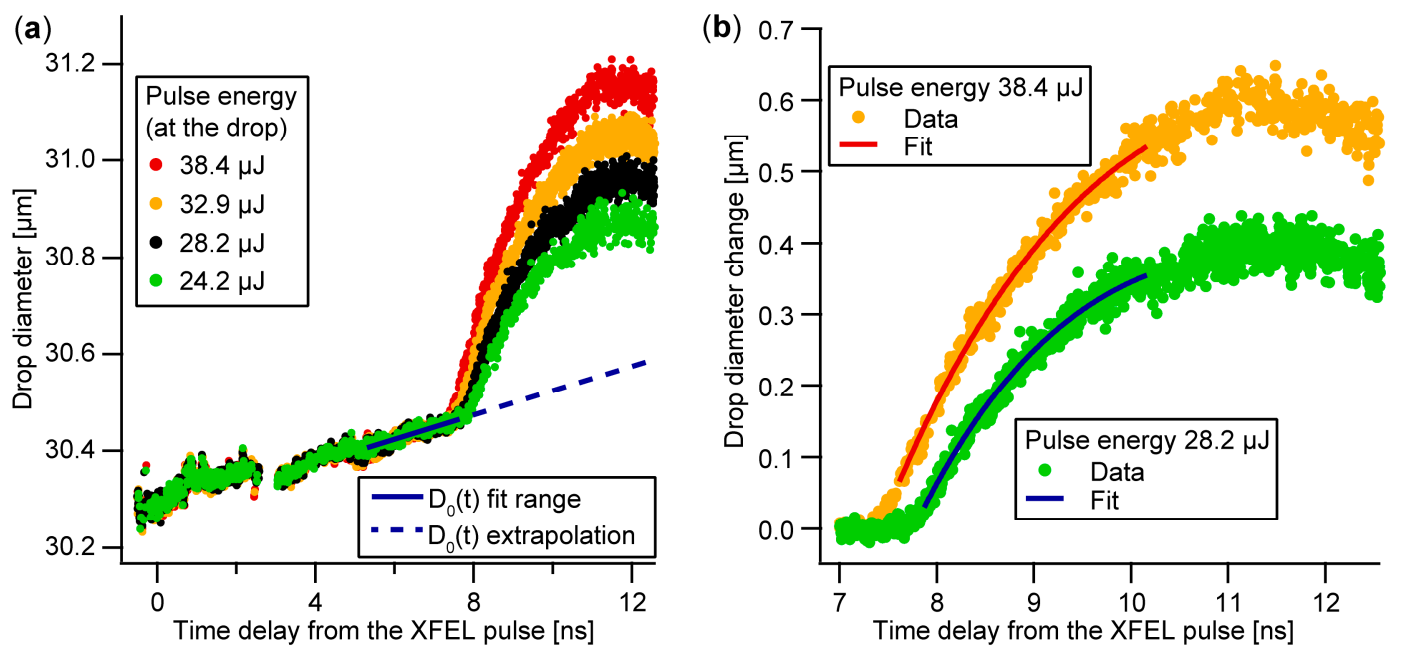

Figure A1. Raw data from the experimental run that exhibited the largest drifts in the initial drop diameter, and the corresponding fits. (a) Drop diameter measurements. For clarity, only four of the 10 extracted data sets are shown. (b) The change in drop diameter during the shock reflection, $D_{D}(t)-\mathrm{D}_{0}(t)$, for two of the data sets shown in the left panel.

The diameter data is fitted for time delays that exclude data within a few hundred picoseconds of the shock arrival, see Figures $3 \mathrm{~b}$ and A1b. In a perfectly aligned drop the slope of the diameter data should change instantaneously upon the shock arrival, but the recorded data shows a gradual increase in slope due to the jitter and the systematic misalignment of the drops. The droplet centers are aligned with the beam to better than $1 \mu \mathrm{m}$, but even misalignments of $0.1 \mu \mathrm{m}$ will make the shocks arrive at measurably different times at the two equatorial surfaces, leading to smearing of the data recorded at delays within a few hundred picoseconds of the shock arrival time in a perfectly centered drop. To choose the first data point for fitting, we first identified the earliest-delay point from where all measurements at longer delays were larger than the peak (diameter-subtracted) diameter of undisturbed drops. These peak values, caused by drop diameter noise, were typically around $20 \mathrm{~nm}$ for the $\sim 30-\mu \mathrm{m}$ drops. For runs collected at the smallest pulse energies, this first point was located after the gradually rising slope and was thus chosen as the first point for fitting. At larger pulse energies, we empirically increased the threshold value up to $100 \mathrm{~nm}$ to avoid fitting data near the shock arrival time. The true rise times of the shock waves were too short to be measured. Since the run in which the drops were best aligned to the XFEL beam had an experimental rise time around $75 \mathrm{ps,} \mathrm{we} \mathrm{estimate}$ that the true rise time was shorter than 75 ps.

\section{Appendix B}

Determination of the waveforms of decaying cylindrical shocks from the surface velocity data. Due to the cylindrical symmetry and the pressure decay, a basic determination of the shock pressure from the shock velocity using the Rankine-Hugoniot relations for plane waves may not be accurate $[42,55]$. For the determination of the initial pressure jump, Equation (3), which is derived for a plane shock, remains appropriate because the shock thickness is much smaller than the curvature of the shock. Using the upper estimate of the shock rise time from experimental data, $75 \mathrm{ps,}$ and a typical shock velocity of $2 \mathrm{~km} / \mathrm{s}$ for the present data, we estimate a shock thickness smaller than $150 \mathrm{~nm}$, which is less than 0.01 of the shock radius of curvature of $\sim 15 \mu \mathrm{m}$ at the equatorial surface of a $\sim 30 \mu \mathrm{m}$ diameter drop. We also note that the assumption of a particle velocity equal to half of the surface velocity [33,56], which is implicit in Equation (2), is likely to be appropriate because the peak shock pressures are below $1 \mathrm{GPa}$ and no phase transitions occur up to this pressure. 
The fast decay of the shock requires a specific analysis for the determination of the pressure waveform, and here we chose a method based on Lagrangian wave analysis [41,42] using Fowles and William's phase velocity concept [40]. The main result is Equation (4), which allows the numerical integration of pressure from its peak value using the particle velocity history [57]. For plane shocks in a material that is isotropic and does not exhibit phase transitions, the phase velocity $C_{C}\left[P_{S}(t)\right]$ is equal to the Lagrangian phase velocity $c_{L}\left[P_{S}(t)\right]$, which is equal to the speed of sound in the compressed material multiplied by the ratio of the compressed material density and the zero-pressure density [38].

In a cylindrically symmetric wave, the phase velocity is different from the speed of sound even for linear, low-amplitude pressure waves. Basically, this occurs because in a cylindrically symmetric system the wave equation has outgoing wave solutions that are not plane waves, but are given by [58] $P(r, t)=\mathrm{A} \mathrm{H}_{0}{ }^{(2)}(\mathrm{k} r) \exp (\mathrm{i} \omega t)$ where $\mathrm{A}$ is the wave amplitude and $\mathrm{H}_{0}{ }^{(2)}$ is a Hankel function (a linear combination of Bessel functions). Far from the source $(\mathrm{k} r \gg 1), \mathrm{H}_{0}{ }^{(2)}(\mathrm{k} r)$ is approximately equal to $(2 / \pi)(\mathrm{k} r)^{-1 / 2} \exp (-\mathrm{ik} r+\pi / 4)$ and the phase velocity $\mathrm{C}_{\text {phase }}$ is the same as in a harmonic plane wave, $\mathrm{C}_{\text {phase }}=\omega / \mathrm{k}$. However, near the origin $(\mathrm{k} r<\sim 10)$ the distance between the consecutive roots of the real part of the Hankel function is larger than $\pi / \mathrm{k}$, thus the 'wavelength' of a cylindrical wave is longer than $2 \pi / k$, and correspondingly near the symmetry axis $C_{\text {phase }}$ is larger than $\omega / \mathrm{k}$.

We derived the phase velocity to be used in Equation (4), $\mathrm{C}_{C}\left[P_{S}(t)\right]$, by imposing that (i) both mass and momentum were conserved in the cylindrical wave and (ii) the pressure and density were related by an adiabatic equation of state.

For the equation of state of water, we used a modified Tait equation of state (see the Supplemental Material of Reference [16]):

$$
\left(P+\mathrm{P}_{\infty}\right) /\left(\mathrm{P}_{0}+\mathrm{P}_{\infty}\right)=\left(\rho / \rho_{0}\right)^{\gamma},
$$

where $P$ and $\rho$ are the absolute pressure and the density, $\mathrm{P}_{0}=1.01325 \times 10^{5} \mathrm{~Pa}$ and $\rho_{0}=996.7 \mathrm{~kg} / \mathrm{m}^{3}$ are the pressure and density before the shock (i.e., water at atmospheric pressure and $26.2^{\circ} \mathrm{C}$ ). Equation (A1) is referenced to the liquid density $\rho_{0}$ at atmospheric pressure, which makes it more convenient to use than the more common form of the Tait equation [59], which is referenced to the liquid density at zero pressure. We used a value $\gamma=7.15$, as employed in other studies [16]. The value of the constant $\mathrm{P}_{\infty}, \mathrm{P}_{\infty}$ $=3.1258 \times 10^{8} \mathrm{~Pa}$, was determined by requiring that the speed of sound $\mathrm{c}_{0}$ at $26.2{ }^{\circ} \mathrm{C}, \mathrm{c}_{0}=1497.7 \mathrm{~m} / \mathrm{s}$, is equal to the speed of sound $c_{\text {eos }}$ calculated from the equation of state (Equation (A1)):

$$
\mathrm{ceos}^{2}=(\partial P / \partial \rho)_{S}=\gamma\left(P+P_{\infty}\right) / \rho,
$$

Following Fowles' derivation of the conservation relations for stress waves in spherical coordinates [43], we derived the conservation laws for density and pressure in cylindrical coordinates:

$$
\begin{gathered}
\mathrm{d} \rho=\left(\rho^{2} r\right) /\left(\rho_{0} \mathrm{r}_{0} \mathrm{C}_{\mathrm{u}}\right) \mathrm{d} u+(\rho u) / r \mathrm{~d} t, \\
\mathrm{~d} P=\left(\rho_{0} \mathrm{r}_{0} \mathrm{C}_{\mathrm{P}}\right) / r \mathrm{~d} u,
\end{gathered}
$$

where $\rho, P, u$ and $r$ are the density, pressure, particle velocity, and radial position of the Lagrangian fluid element, $\rho_{0}, \mathrm{P}_{0}$, and $\mathrm{r}_{0}$ the corresponding values before the shock arrival. The two phase velocities [40] are defined by $\mathrm{C}_{\mathrm{u}}=(\partial h / \partial t)_{\mathrm{u}}$ and $\mathrm{C}_{\mathrm{P}}=(\partial h / \partial t)_{\mathrm{P}}$, where $h$ is the Lagrangian coordinate of the fluid element. Since the flow of the liquid after the shock discontinuity is adiabatic, and since no phase transitions occur during flow, we assumed that the phase velocities are equal: $C_{u}=C_{P}=C_{C}(P)$.

At any given pressure $P$, Equation (A2) can be used to derive the density variation $\mathrm{d} \rho_{\text {eos }}=\mathrm{d} P / \mathrm{c}_{\text {eos }}{ }^{2}$ corresponding to the differential change in pressure $\mathrm{d} P$ given by Equation (A4). The condition that the change in density should be the same when calculated by Equation (A3) as when calculated from Equation (A4) via the equation of state (i.e., $\mathrm{d} \rho=\mathrm{d} \rho_{\text {eos }}$ ) leads to a quadratic equation in $\mathrm{C}_{\mathrm{C}}(P)$, from which we calculated the phase velocity.

Finally, the shock waveforms $P_{S}(t)$ were calculated by numerical integration of Equation (4) from $t_{\text {shock }}$ until $t_{\text {acoustic }}$. The initial pressure was calculated using Equation (3). The analytical fit of the 
surface position, see Equations (1) and (2), was used to determine the particle position $r$, the particle velocity $u$, and its derivative $\mathrm{d} u / \mathrm{d} t$. The phase velocity $C_{C}\left(P_{S}(t)\right)$ was calculated at each time step using the computed values of $r, u$, and $\mathrm{d} u / \mathrm{d} t$ at the same time step, and the pressure $P_{S}$ at the previous time step. The numerical integration was done in 1000 time steps using a computer code written in Matlab (MATLAB R2018a, The MathWorks, Inc., Natick, MA, USA).

\section{Appendix C}

The fitting parameters for the particle velocity data for all measurements are given in Table A1, along with the initial shock wave pressures. For droplets with 29.9-30.5 $\mu$ m diameters, 79 measurements were done for 55 distinct XFEL pulse energies. For droplets with $60.5 \mu \mathrm{m}$ diameters, 11 measurements were done for 11 distinct XFEL pulse energies. The standard deviations given in Table A1 are the standard deviations of the fit parameters as reported by the software used for fitting (Igor Pro 8, Wavemetrics Inc.) and do not account for systematic errors, such as second-order effects of the drifts in the undisturbed drop diameter.

Table A1. Measurement conditions, fit parameters for particle velocities (see Equation (1)), and the initial shock wave pressures $P_{S}\left(t_{\text {shock }}\right)$. $D_{\text {drop }}$ is the undisturbed drop diameter just before the arrival of the shock wave, and $E_{x r a y}$ is the energy of the X-ray pulse arriving at the droplets. The standard deviations represent the statistical uncertainties of the fit parameters.

\begin{tabular}{|c|c|c|c|c|c|c|c|c|}
\hline$D_{\text {drop }}[\mu \mathrm{m}]$ & $E_{\text {xray }}[\mu \mathrm{J}]$ & $\mathrm{V}_{0}[\mathrm{~m} / \mathrm{s}]$ & $\sigma_{\mathrm{v} 0}[\mathrm{~m} / \mathrm{s}]$ & $\mathbf{t}_{\text {shock }}[\mathrm{ns}]$ & $\sigma_{\mathrm{ts}}[\mathrm{ns}]$ & $\mathbf{t}_{\text {decay }}[\mathrm{ns}]$ & $\sigma_{\mathrm{td}}[\mathrm{ns}]$ & $P_{S}\left(\mathrm{t}_{\text {shock }}\right)[\mathrm{MPa}]$ \\
\hline 30.2 & 7.1 & 113 & 16 & 8.991 & 0.044 & 1.82 & 0.43 & 44 \\
\hline 30.2 & 7.4 & 123 & 15 & 8.943 & 0.039 & 1.72 & 0.34 & 48 \\
\hline 30.2 & 7.8 & 119 & 12 & 8.883 & 0.034 & 2.03 & 0.36 & 46 \\
\hline 30.2 & 8.2 & 121 & 15 & 8.839 & 0.047 & 2.11 & 0.43 & 47 \\
\hline 30.2 & 8.7 & 131 & 12 & 8.819 & 0.033 & 2.05 & 0.31 & 51 \\
\hline 30.2 & 9.1 & 127 & 10 & 8.765 & 0.032 & 2.55 & 0.39 & 50 \\
\hline 30.2 & 9.6 & 142 & 11 & 8.732 & 0.033 & 2.27 & 0.29 & 56 \\
\hline 30.2 & 10.1 & 139 & 9 & 8.677 & 0.028 & 2.61 & 0.32 & 54 \\
\hline 30.2 & 10.7 & 142 & 7 & 8.638 & 0.019 & 2.81 & 0.31 & 56 \\
\hline 30.2 & 11.2 & 145 & 11 & 8.592 & 0.030 & 2.97 & 0.50 & 57 \\
\hline 30.2 & 15.3 & 230 & 7 & 8.242 & 0.012 & 2.09 & 0.08 & 92 \\
\hline 30.5 & 15.3 & 193 & 7 & 8.339 & 0.016 & 2.66 & 0.15 & 77 \\
\hline 30.5 & 16.1 & 202 & 6 & 8.289 & 0.015 & 2.75 & 0.14 & 81 \\
\hline 30.2 & 16.1 & 212 & 6 & 8.139 & 0.011 & 2.72 & 0.12 & 85 \\
\hline 30.5 & 16.1 & 201 & 7 & 8.321 & 0.015 & 2.56 & 0.14 & 80 \\
\hline 30.5 & 16.9 & 207 & 5 & 8.225 & 0.011 & 2.88 & 0.12 & 83 \\
\hline 30.5 & 16.9 & 203 & 5 & 8.265 & 0.012 & 2.73 & 0.11 & 81 \\
\hline 30.2 & 16.9 & 206 & 5 & 8.069 & 0.010 & 3.14 & 0.12 & 82 \\
\hline 30.5 & 17.8 & 208 & 6 & 8.203 & 0.014 & 2.86 & 0.13 & 83 \\
\hline 30.2 & 17.8 & 232 & 5 & 8.061 & 0.011 & 2.85 & 0.10 & 93 \\
\hline 30.5 & 17.8 & 218 & 5 & 8.178 & 0.011 & 2.93 & 0.11 & 87 \\
\hline 30.5 & 18.7 & 221 & 4 & 8.102 & 0.010 & 3.17 & 0.11 & 88 \\
\hline 30.2 & 18.7 & 235 & 4 & 7.981 & 0.009 & 3.06 & 0.09 & 95 \\
\hline 30.5 & 18.7 & 222 & 4 & 8.163 & 0.008 & 2.88 & 0.09 & 89 \\
\hline 30.2 & 19.7 & 238 & 4 & 7.910 & 0.008 & 3.34 & 0.09 & 96 \\
\hline 30.5 & 19.7 & 234 & 5 & 8.071 & 0.011 & 3.30 & 0.12 & 94 \\
\hline 30.5 & 19.7 & 229 & 4 & 8.124 & 0.008 & 3.02 & 0.09 & 92 \\
\hline 30.5 & 20.8 & 254 & 4 & 8.026 & 0.007 & 3.18 & 0.08 & 103 \\
\hline 30.5 & 20.8 & 248 & 4 & 8.092 & 0.008 & 2.94 & 0.07 & 100 \\
\hline 30.2 & 20.8 & 237 & 4 & 7.839 & 0.008 & 3.76 & 0.11 & 95 \\
\hline 30.2 & 21.8 & 242 & 4 & 7.755 & 0.009 & 4.07 & 0.14 & 98 \\
\hline 30.5 & 21.8 & 250 & 4 & 7.947 & 0.009 & 3.63 & 0.11 & 101 \\
\hline 30.5 & 21.8 & 255 & 4 & 8.037 & 0.007 & 3.11 & 0.07 & 103 \\
\hline 30.2 & 23.0 & 266 & 5 & 7.749 & 0.010 & 3.92 & 0.13 & 108 \\
\hline 30.5 & 23.0 & 264 & 4 & 7.981 & 0.007 & 3.27 & 0.08 & 107 \\
\hline 30.5 & 23.0 & 264 & 5 & 7.907 & 0.010 & 3.68 & 0.13 & 107 \\
\hline 30.2 & 24.2 & 273 & 7 & 7.690 & 0.013 & 4.18 & 0.20 & 111 \\
\hline 30.5 & 24.2 & 292 & 6 & 7.870 & 0.012 & 3.44 & 0.12 & 120 \\
\hline 30.5 & 24.2 & 240 & 8 & 7.919 & 0.018 & 3.43 & 0.20 & 97 \\
\hline
\end{tabular}


Table A1. Cont.

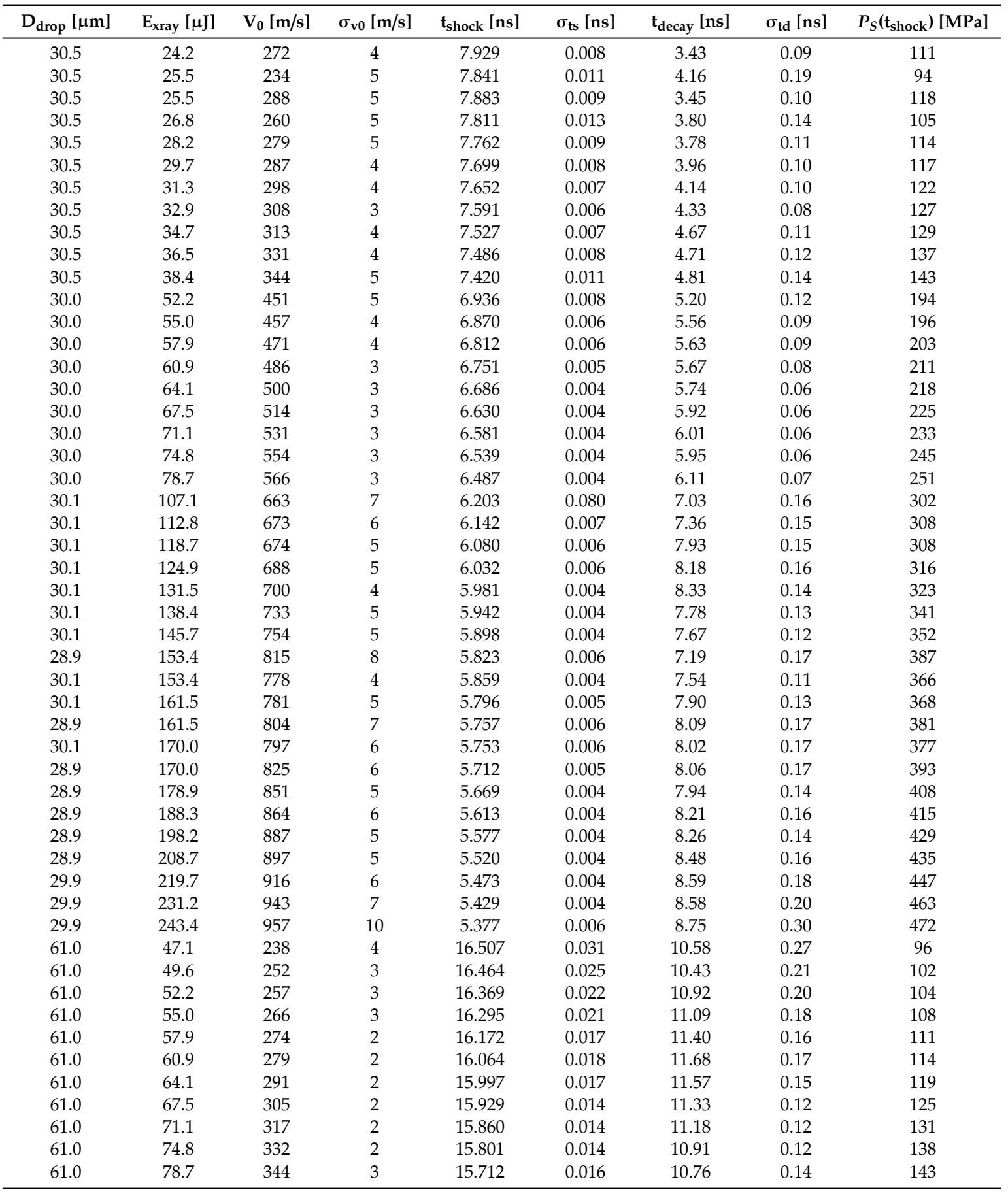

\section{References}

1. Vogel, A.; Venugopalan, V. Mechanisms of Pulsed Laser Ablation of Biological Tissues. Chem. Rev. 2003, 103, 577-644. [CrossRef]

2. Vogel, A.; Noack, J.; Huttman, G.; Paltauf, G. Mechanisms of femtosecond laser nanosurgery of cells and tissues. Appl. Phys. B 2005, 81, 1015-1047. [CrossRef]

3. Chichkov, B.N.; Momma, C.; Nolte, S.; von Alvensleben, F.; Tunnermann, A. Femtosecond, picosecond and nanosecond laser ablation of solids. Appl. Phys. A 1996, 63, 109-115. [CrossRef]

4. Nolte, S.; Momma, C.; Jacobs, H.; Tunnermann, A.; Chichkov, B.N.; Wellegehausen, B.; Welling, H. Ablation of metals by ultrashort laser pulses. J. Opt. Soc. Am. B Opt. Phys. 1997, 14, 2716-2722. [CrossRef] 
5. Liu, X.; Du, D.; Mourou, G. Laser ablation and micromachining with ultrashort laser pulses. IEEE J. Quantum Electron. 1997, 33, 1706-1716. [CrossRef]

6. Yang, G.W. Laser ablation in liquids: Applications in the synthesis of nanocrystals. Prog. Mater. Sci. 2007, 52, 648-698. [CrossRef]

7. Vogel, A.; Lauterborn, W. Acoustic Transient Generation by Laser-Produced Cavitation Bubbles near Solid Boundaries. J. Acoust. Soc. Am. 1988, 84, 719-731. [CrossRef]

8. Vogel, A.; Noack, J. Shock-wave energy and acoustic energy dissipation after laser-induced breakdown. In Laser-Tissue Interaction IX; International Society for Optics and Photonics: Bellingham, WA, USA, 1998; pp. 180-189.

9. Anderhol, N.C. Laser-generated stress waves. Appl. Phys. Lett. 1970, 16, 113-115. [CrossRef]

10. Smith, R.F.; Eggert, J.H.; Jeanloz, R.; Duffy, T.S.; Braun, D.G.; Patterson, J.R.; Rudd, R.E.; Biener, J.; Lazicki, A.E.; Hamza, A.V.; et al. Ramp compression of diamond to five terapascals. Nature 2014, 511, 330-333. [CrossRef]

11. Meyers, M.A.; Gregori, F.; Kad, B.K.; Schneider, M.S.; Kalantar, D.H.; Remington, B.A.; Ravichandran, G.; Boehly, T.; Wark, J.S. Laser-induced shock compression of monocrystalline copper: Characterization and analysis. Acta Mater. 2003, 51, 1211-1228. [CrossRef]

12. Carlson, G.A. Dynamic tensile strength of mercury. J. Appl. Phys. 1975, 46, 4069-4070. [CrossRef]

13. Fortov, V.E.; Kostin, V.V.; Eliezer, S. Spallation of metals under laser irradiation. J. Appl. Phys. 1991, 70, 4524-4531. [CrossRef]

14. Tamura, H.; Kohama, T.; Kondo, K.; Yoshida, M. Femtosecond-laser-induced spallation in aluminum. J. Appl. Phys. 2001, 89, 3520-3522. [CrossRef]

15. Paltauf, G.; Schmidt-Kloiber, H. Microcavity dynamics during laser-induced spallation of liquids and gels. Appl. Phys. A 1996, 62, 303-311. [CrossRef]

16. Ando, K.; Liu, A.Q.; Ohl, C.D. Homogeneous Nucleation in Water in Microfluidic Channels. Phys. Rev. Lett. 2012, 109, 044501. [CrossRef]

17. Stan, C.A.; Willmott, P.R.; Stone, H.A.; Koglin, J.E.; Liang, M.; Aquila, A.L.; Robinson, J.S.; Gumerlock, K.L.; Blaj, G.; Sierra, R.G.; et al. Negative Pressures and Spallation in Water Drops Subjected to Nanosecond Shock Waves. J. Phys. Chem. Lett. 2016, 7, 2055-2062. [CrossRef]

18. Emma, P.; Akre, R.; Arthur, J.; Bionta, R.; Bostedt, C.; Bozek, J.; Brachmann, A.; Bucksbaum, P.; Coffee, R.; Decker, F.J.; et al. First lasing and operation of an ångstrom-wavelength free-electron laser. Nat. Photon. 2010, 4, 641-647. [CrossRef]

19. Ishikawa, T.; Aoyagi, H.; Asaka, T.; Asano, Y.; Azumi, N.; Bizen, T.; Ego, H.; Fukami, K.; Fukui, T.; Furukawa, Y.; et al. A compact X-ray free-electron laser emitting in the sub-ångström region. Nat. Photon. 2012, 6, 540-544. [CrossRef]

20. Mimura, H.; Yumoto, H.; Matsuyama, S.; Koyama, T.; Tono, K.; Inubushi, Y.; Togashi, T.; Sato, T.; Kim, J.; Fukui, R.; et al. Generation of 10^20 Wcm^-2 hard X-ray laser pulses with two-stage reflective focusing system. Nat. Commun. 2014, 5, 3539. [CrossRef]

21. Vinko, S.M.; Ciricosta, O.; Cho, B.I.; Engelhorn, K.; Chung, H.K.; Brown, C.R.D.; Burian, T.; Chalupsky, J.; Falcone, R.W.; Graves, C.; et al. Creation and diagnosis of a solid-density plasma with an X-ray free-electron laser. Nature 2012, 482, 59-62. [CrossRef]

22. Yoneda, H.; Inubushi, Y.; Yabashi, M.; Katayama, T.; Ishikawa, T.; Ohashi, H.; Yumoto, H.; Yamauchi, K.; Mimura, H.; Kitamura, H. Saturable absorption of intense hard X-rays in iron. Nat. Commun. 2014, 5, 5080. [CrossRef] [PubMed]

23. Beyerlein, K.R.; Jonsson, H.O.; Alonso-Mori, R.; Aquila, A.; Barty, S.; Barty, A.; Bean, R.; Koglin, J.E.; Messerschmidt, M.; Ragazzon, D.; et al. Ultrafast nonthermal heating of water initiated by an X-ray Free-Electron Laser. Proc. Natl. Acad. Sci. USA 2018, 115, 5652-5657. [CrossRef] [PubMed]

24. Stan, C.A.; Milathianaki, D.; Laksmono, H.; Sierra, R.G.; McQueen, T.A.; Messerschmidt, M.; Williams, G.J.; Koglin, J.E.; Lane, T.J.; Hayes, M.J.; et al. Liquid explosions induced by X-ray laser pulses. Nat. Phys. 2016, 12, 966-971. [CrossRef]

25. Chapman, H.N.; Fromme, P.; Barty, A.; White, T.A.; Kirian, R.A.; Aquila, A.; Hunter, M.S.; Schulz, J.; DePonte, D.P.; Weierstall, U.; et al. Femtosecond X-ray protein nanocrystallography. Nature 2011, 470, $73-77$. [CrossRef]

26. Altarelli, M. The European X-ray Free-Electron Laser: Toward an ultra-bright, high repetition-rate x-ray source. High Power Laser Sci. 2015, 3, e18. [CrossRef] 
27. Grünbein, M.L.; Bielecki, J.; Gorel, A.; Stricker, M.; Bean, R.; Cammarata, M.; Doerner, K.; Fröhlich, L.; Hartmann, L.; Hauf, S.; et al. Megahertz Data Collection from Protein Microcrystals at an X-ray Free-Electron Laser. Nat. Commun. 2018, 9, 3847. [CrossRef]

28. Wiedorn, M.O.; Oberthür, D.; Bean, R.; Schubert, R.; Werner, N.; Abbey, B.; Aepfelbacher, M.; Adriano, A.; Allahgholi, A.; Al-Qudami, N.; et al. Megahertz serial crystallography. Nat. Commun. 2018, 9, 4025. [CrossRef]

29. Yefanov, O.; Oberthür, D.; Bean, R.; Wiedorn, M.O.; Knoska, J.; Pena, G.; Awel, S.; Gumprecht, L.; Domaracky, M.; Sarrou, I.; et al. Evaluation of serial crystallographic structure determination within megahertz pulse trains. Struct. Dyn. 2019, 6, 064702. [CrossRef]

30. Blaj, G.; Liang, M.N.; Aquila, A.L.; Willmott, P.R.; Koglin, J.E.; Sierra, R.G.; Robinson, J.S.; Boutet, S.; Stan, C.A. Generation of high-intensity ultrasound through shock propagation in liquid jets. Phys. Rev. Fluids 2019, 4, 043401. [CrossRef]

31. Tono, K.; Togashi, T.; Inubushi, Y.; Sato, T.; Katayama, T.; Ogawa, K.; Ohashi, H.; Kimura, H.; Takahashi, S.; Takeshita, K.; et al. Beamline, experimental stations and photon beam diagnostics for the hard x-ray free electron laser of SACLA. New J. Phys. 2013, 15, 083035. [CrossRef]

32. Yumoto, H.; Mimura, H.; Koyama, T.; Matsuyama, S.; Tono, K.; Togashi, T.; Inubushi, Y.; Sato, T.; Tanaka, T.; Kimura, T.; et al. Focusing of X-ray free-electron laser pulses with reflective optics. Nat. Photon. 2013, 7, 43-47. [CrossRef]

33. Walsh, J.M.; Christian, R.H. Equation of State of Metals from Shock Wave Measurements. Phys. Rev. 1955, 97, 1544-1556. [CrossRef]

34. Henderson, L.F. On the refraction of shock waves. J. Fluid Mech. 1989, 198, 365-386. [CrossRef]

35. Tono, K.; Kudo, T.; Yabashi, M.; Tachibana, T.; Feng, Y.P.; Fritz, D.; Hastings, J.; Ishikawa, T. Single-shot beam-position monitor for x-ray free electron laser. Rev. Sci. Instrum. 2011, 82, 023108. [CrossRef] [PubMed]

36. Kudo, T.; Tono, K.; Yabashi, M.; Togashi, T.; Sato, T.; Inubushi, Y.; Omodani, M.; Kirihara, Y.; Matsushita, T.; Kobayashi, K.; et al. A photodiode amplifier system for pulse-by-pulse intensity measurement of an x-ray free electron laser. Rev. Sci. Instrum. 2012, 83, 043108. [CrossRef] [PubMed]

37. Kato, M.; Tanaka, T.; Kurosawa, T.; Saito, N.; Richter, M.; Sorokin, A.A.; Tiedtke, K.; Kudo, T.; Tono, K.; Yabashi, M.; et al. Pulse energy measurement at the hard x-ray laser in Japan. Appl. Phys. Lett. 2012, 101, 023503. [CrossRef]

38. Grady, D. Shocks and structured waves. In Physics of Shock and Impact; IOP Publishing Ltd.: Bristol, UK, 2017.

39. Nagayama, K.; Mori, Y.; Shimada, K.; Nakahara, M. Shock Hugoniot compression curve for water up to 1 GPa by using a compressed gas gun. J. Appl. Phys. 2002, 91, 476-482. [CrossRef]

40. Fowles, R.; Williams, R.F. Plane Stress Wave Propagation in Solids. J. Appl. Phys. 1970, 41, 360-363. [CrossRef]

41. Aidun, J.B.; Gupta, Y.M. Analysis of Lagrangian Gauge Measurements of Simple and Nonsimple Plane-Waves. J. Appl. Phys. 1991, 69, 6998-7014. [CrossRef]

42. Grady, D. Shock wave analysis and applications. In Physics of Shock and Impact; IOP Publishing Ltd.: Bristol, UK, 2017.

43. Fowles, R. Conservation Relations for Spherical and Cylindrical Stress Waves. J. Appl. Phys. 1970, 41, 2740-2741. [CrossRef]

44. Rice, M.H.; Walsh, J.M. Equation of State of Water to 250 Kilobars. J. Chem. Phys. 1957, 26, 824-830. [CrossRef]

45. Lyu, X.X.; Pan, S.C.; Hu, X.Y.; Adams, N.A. Numerical investigation of homogeneous cavitation nucleation in a microchannel. Phys. Rev. Fluids 2018, 3, 064303. [CrossRef]

46. Veysset, D.; Gutierrez-Hernandez, U.; Dresselhaus-Cooper, L.; De Colle, F.; Kooi, S.; Nelson, K.A.; Quinto-Su, P.A.; Pezeril, T. Single-bubble and multibubble cavitation in water triggered by laser-driven focusing shock waves. Phys. Rev. E 2018, 97, 053112. [CrossRef] [PubMed]

47. Paula, T.; Adami, S.; Adams, N.A. Analysis of the early stages of liquid-water-drop explosion by numerical simulation. Phys. Rev. Fluids 2019, 4, 044003. [CrossRef]

48. Cai, Y.; Wu, H.A.; Luo, S.N. Spall strength of liquid copper and accuracy of the acoustic method. J. Appl. Phys. 2017, 121, 105901. [CrossRef]

49. Barker, L.; Hollenbach, R. Laser interferometer for measuring high velocities of any reflecting surface. J. Appl. Phys. 1972, 43, 4669-4675. [CrossRef]

50. Menezes, V.; Hosseini, H.; Moosavi-Nejad, S.; Irimpan, K.J.; Akiyama, H. Motion of free-surface of shock-compressed water on emergence of rarefaction. Appl. Phys. Lett. 2015, 107, 143701. [CrossRef] 
51. Li, Z.G.; Xiong, S.; Chin, L.K.; Ando, K.; Zhang, J.B.; Liu, A.Q. Water's tensile strength measured using an optofluidic chip. Lab Chip 2015, 15, 2158-2161. [CrossRef]

52. Savitzky, A.; Golay, M.J.E. Smoothing and Differentiation of Data by Simplified Least Squares Procedures. Anal. Chem. 1964, 36, 1627-1639. [CrossRef]

53. Friedlander, F.G. The Diffraction of Sound Pulses 1. Diffraction by a Semi-Infinite Plane. Proc. R. Soc. Lond. Ser. A 1946, 186, 322-344.

54. Dewey, J.M. The Friedlander Equations. In Blast Effects: Physical Properties of Shock Waves, 1st ed.; Sochet, I., Ed.; Springer: Cham, Switzerland, 2018; pp. 37-55.

55. Krehl, P.O.K. The classical Rankine-Hugoniot jump conditions, an important cornerstone of modern shock wave physics: Ideal assumptions vs. reality. Eur. Phys. J. H 2015, 40, 159-204. [CrossRef]

56. Luo, S.N.; Han, L.B.; Xie, Y.; An, Q.; Zheng, L.Q.; Xia, K.W. The relation between shock-state particle velocity and free surface velocity: A molecular dynamics study on single crystal Cu and silica glass. J. Appl. Phys. 2008, 103, 093530. [CrossRef]

57. Rothman, S.D.; Davis, J.P.; Maw, J.; Robinson, C.M.; Parker, K.; Palmer, J. Measurement of the principal isentropes of lead and lead-antimony alloy to similar to $400 \mathrm{kbar}$ by quasi-isentropic compression. J. Phys. D Appl. Phys. 2005, 38, 733-740. [CrossRef]

58. Kinsler, L.E.; Frey, A.R.; Coppens, A.B.; Sanders, J.V. The acoustic wave equation and simple solutions. In Fundamentals of Acoustics, 4th ed.; John Wiley \& Sons, Inc.: New York, NY, USA, 1999; pp. 113-148.

59. Thompson, P.A. Compressible-Fluid Dynamics; McGraw-Hill: New York, NY, USA, 1972; pp. 102-103.

(C) 2020 by the authors. Licensee MDPI, Basel, Switzerland. This article is an open access article distributed under the terms and conditions of the Creative Commons Attribution (CC BY) license (http://creativecommons.org/licenses/by/4.0/). 